\title{
Effect of diclofenac on TNFa and HIF1a levels in rat supraspinatus tendon repair
}

\author{
Haluk Çabuk ${ }^{1}$ \\ Fatmagül Kuşku Çabuk ${ }^{2}$ \\ 1 Department of Orthopaedics and Traumatology \\ Okmeydani Training and Research Hospital, Istanbul, \\ Turkey \\ 2 Department of Pathology, Bakırköy Dr. Sadi Konuk \\ Training and Research Hospital, Istanbul, Turkey
}

\author{
Corresponding author: \\ Haluk Çabuk \\ Department of Orthopaedics and Traumatology \\ Okmeydani Training and Research Hospital \\ Cumhuriyet cad. camlica sok. 4 A, no 13 Göktürk/Eyüp \\ 34077 Istanbul, Turkey \\ E-mail: halukcabuk@hotmail.com
}

\section{Summary}

Introduction: Tendon healing is influenced by proinflammatory cytokines such as tumour necrosis factor alpha (TNFa) and hypoxia-induced factor 1 alpha (HIF1a). We hypothese that diclofenac effects on HIF1a and TNFa levels during healing process.

Methods: A supraspinatus tear model was created and repaired to its anatomic footprint with $\mathbf{4 2}$ male Winstar rats. Rats were randomized to study and control group. Diclofenac $1 \mathrm{mg} / \mathrm{kg} / \mathrm{day}$ was administered to the study group in a subcutaneous way. The rats were killed at weeks 1, 3 and 6, and seven rats from each group underwent immunohistochemical examination with TNFa and HIF1a.

Results: HIF1a was highest in the first week in both diclofenac and control group. HIF1a levels decreased significantly at the $6^{\text {th }}$ week in the control group $(p=0.011)$ but in diclofenac group this decrease was observed at the $3^{\text {rd }}$ week $(p=0.024)$. TNFa levels decrease significantly at $3^{\text {rd }}$ week in both groups $(p<0.001)$, but in diclofenac group TNFa level was high at $6^{\text {th }}$ week compared to $3^{\text {rd }}$ week $(p=0.051)$.

Conclusion: Diclofenac could have effect on HIF1a and TNFa which are important in different phases of tendon healing.

Level of evidence: basic science study.

KEY WORDS: tumour necrosis factor-alpha, rotator cuff, diclofenac, hypoxia-inducible factor 1 alpha, inflammation.

\section{Introduction}

Rotator cuff tears are a major cause of shoulder pain and dysfunction. Despite surgical treatment, recurrence rates are very high in massive tears ${ }^{1}$. Early retear rates could be up to $40 \%$ in large tears in one year $^{2}$. Although the size of tear is associated with retear rate, it is mainly a multifactorial process ${ }^{3}$. Hypoxia, ischaemic damage, oxidative stress, hyperthermia, impaired apoptosis, inflammatory mediators, fluoroquinolones, and matrix metalloproteinase imbalance have all been implicated as mechanism of retear and tendinopathies ${ }^{4}$.

NSAIDs are widely prescribed and are used to treat muscle injuries over the years. Among non-steroidal drugs, diclofenac sodium is a non-narcotic pain reliever with the most potent analgesic effect ${ }^{5}$. Diclofenac inhibits the inflammatory response during the tendon healing process via blocking phosphoglandin synthesis from arachidonic acid ${ }^{6}$. Besides NSAIDs could also affect the biomechanical outcomes of tendon healing ${ }^{7}$.

Tendon healing is probably influenced by the presence of pro-inflammatory cytokines such as TNFa. TNFa is one of the most potent proinflammatory cytokine especially in mechanical load and the injurious stimuli ${ }^{8}$. TNFa is a cytokine with diverse functions that is produced by transmembrane protein and cleaved to release soluble protein. TNFa signalling occurs via two structurally related but functionally distinct receptors; TNFR1 induces apoptosis and proinflammatory effects whereas TNFR2 is related to tissue repair, growth-modulating effects, and differentiation $^{9}$.

HIF1a is recognised to control key aspects of inflammation, particularly leucocyte recruitment and subsequent cytokine production ${ }^{10}$. HIF1a regulates tendon regeneration and cell differentiation to tenocytes in tendon healing ${ }^{11}$. HIF1a also induces the expression of several genes which have beneficial effects on the regeneration and protection of many organ systems, including neural tissue, heart, lung, and skeletal muscle $^{12}$. HIF1a is an important transcription factor for angiogenesis. Hypoxia initiates intracellular stabilisation of HIF1a, and HIF complex binds to the hypoxic response element in the promoter region of VEGF gene and induces transcription of genes ${ }^{13}$. Inhibition or ablation of HIF-1 signalling has been shown to reduce angiogenesis and collagen deposition in some fibrotic models ${ }^{14}$.

Our hypothesis was HIF1a and TNFa levels will decrease as the inflammatory phase will end, and as diclofenac blocks the inflammatory response, this de- 
crease in the HIF1a and TNFa levels should be more rapidly.

\section{Materials and methods}

Approval of the Local Ethical Board for Animal Experiments was obtained prior to the study. Principles of laboratory animal care were followed. The study meets the ethical standards of the Journal ${ }^{15}$. A rat model was preferred since the rats have a supraspinatus tendon which passes under an arch similar to that of the human supraspinatus tendon. The study included 42 male Winstar rats weighting 200-300 g. All rats underwent a surgical procedure.

Rats were randomized to the study group $(n=21)$ or the control group $(n=21)$. Then, the groups were divided into three subgroups, representing periods of 1 , 3 and 6 weeks. Daily subcutaneous diclofenac sodium $1 \mathrm{mg} / \mathrm{kg}$ was administered to the rats as same as the human therapeutic dose in the study group. The rats in the control group received no additional treatment after the surgery. At the end of the respective follow-up period, all rats were killed, and 7 rats from each subgroup were used for histopathological analysis.

\section{Surgical procedure}

Prophylaxis was performed using a subcutaneous injection of gentamicin $8 \mathrm{mg} / \mathrm{kg}$ before the surgery. Surgical procedures were carried out under intraperitoneal anaesthesia with a mixture of $50 \mathrm{mg} / \mathrm{kg} \mathrm{Ke}$ tamine- $\mathrm{HCl}$ (Alfamine, Alfasan, TR) and $10 \mathrm{mg} / \mathrm{kg} \mathrm{Xy-}$ lazine $\mathrm{HCl}$ (Alfazyne, Alfasan, TR). The surgical area was cleaned prior to the surgery. All surgical procedures were performed under aseptic conditions. A 1 $\mathrm{cm}$ transverse skin incision was made from the lateral border of the acromion. A portion of the spinodeltoid muscle was detached from the acromion. The acromion was gently retracted to expose the supraspinatus tendon, and the tendon was removed from the attachment point to the tuberculum majus underneath the acromion using the tip of a No. 15 scalpel after placement of a sling suture. The footprint of tendon debrided. Then, the supraspinatus tendon was repaired by suturing it to the original position transosseously with a $4 / 0$ polyester suture. There was no restriction of movement during the post-operative period.

The rats were killed at post-operative weeks 1, 3, and 6 . The humerus and supraspinatus muscle dissected from the rest of the body to form a tendon-humeral unity after exposing the acromial arc without giving any harm to the healing portion of the tendon ${ }^{16}$.

\section{Histology}

After the specimens prepared as a tendon bone unit were fixed in $10 \%$ formaldehyde solution, they were decalcified in $20 \%$ formic acid for 1 day. All specimens were examined in coronal section plane in two $3 \mathrm{~mm}$-thick slices, and embedded in paraffin. Five-micron-thick sections were cut, and stained with hematoxylin and eosin (HE). For examining vascular proliferation, a 30.000 micron square meter was chosen at the tendon bone healing region where maximum vascularity exists. Newly formed vessels were counted and $0-5$ vessels classified as 1, 6-20 classified as 2 and above 21 as $3^{16,17}$.

\section{Immunohistochemical analysis}

For IHC examinations of muscle-tendinosis tissue sections, TNF alfa [4E1]:sc-130349 Santa Cruz biotechnology, Inc Santa Cruz, California, USA and hypoxy-induced factor 1 alpha [(HIF 1alfa: Halpha): sc53546. Santa Cruz biotechnology, Inc Santa Cruz, California, USA] density and intensity of dye uptake of the TNFa antibody was evaluated as described by Khandoga et al. ${ }^{18}$. TNFa was evaluated as no staining (-), slight staining (+) and intense staining (++) (Fig. 1). IHC findings according to the groups were

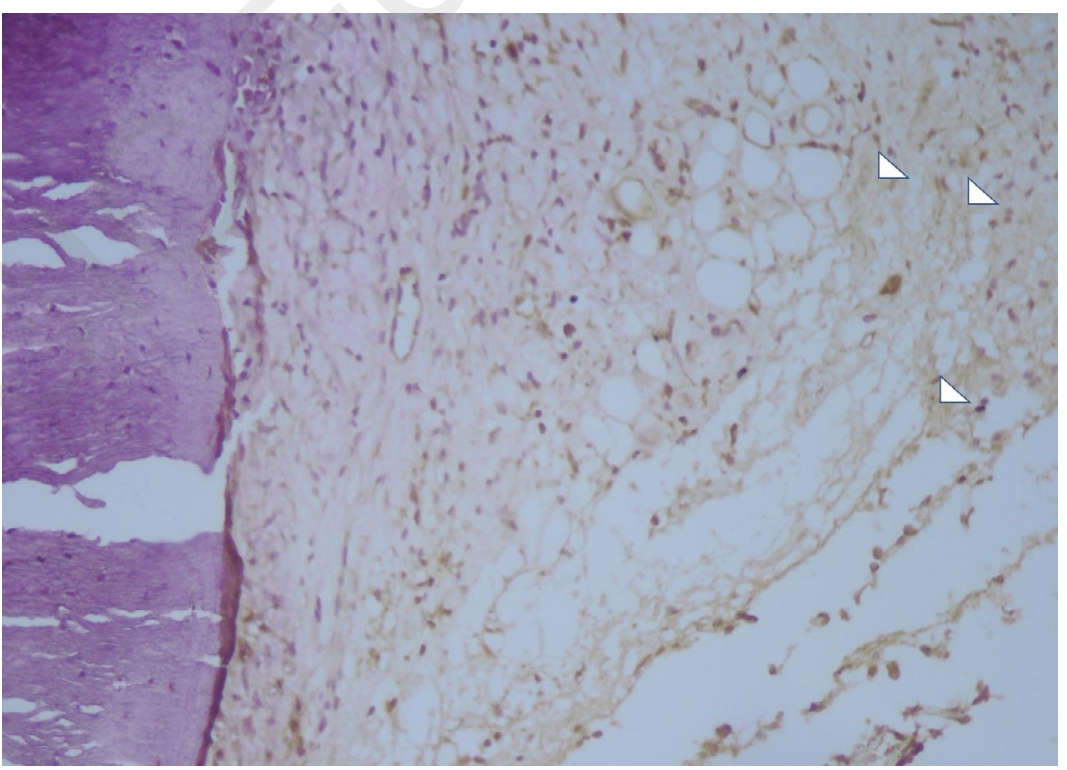

Figure 1. 2+ cytoplasmic staining for TNFa at healing area at 3 weeks. White arrow heads show the cytoplasmic staining of TNFa. 


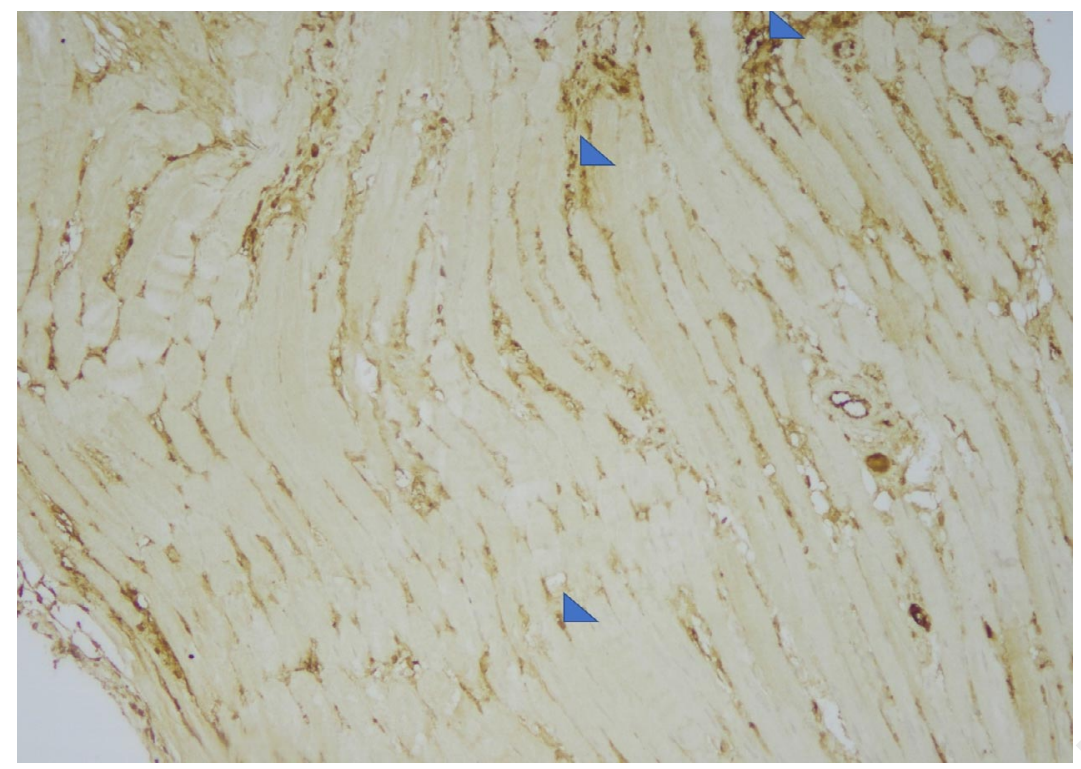

Figure 2. 4+ cytoplasmic and nuclear statining for HIF1a at healing area at 3 weeks. Blue arrow heads show the nuclear staining of HIF1a.

calculated as 0 paints for no staining (-), 1 point for slight staining $(+)$, and 2 points for intense staining $(++)$. HIF-1a was evaluated as (+) for $1-25 \%$ staining, (++) for $26-50 \%$ staining, (+++) for $51-75 \%$ staining and (++++) for $76-100 \%$ staining and they were given degrees as 0, 1, 2, 3 and 4 respectively (Fig. 2). Cytoplasmic staining for TNFa and nuclear and cytoplasmic staining for HIF1a was accepted as positive. All pathologic examinations were performed by pathologists blinded to the groups using light microscopes.

\section{Statistical analysis}

SSPS version 20 software package was used for analysis of data. For histological and immunohistological assessments one way ANOVA test with post-hoc
Bonferroni multiple compressions was used. For correlations of vascular proliferation with HIF1a spearman correlation test was used. A $p$-value $<0.05$ was considered significant.

\section{Results}

There was no loss in rats during the postoperative period. No infection was observed in injection sites and surgical site.

HIF1a was highest in the first week in both diclofenac and control group. HIF1a levels decrease significantly at $6^{\text {th }}$ week in control group $(p=0.011)$ but in the diclofenac group, this decrease was observed at $3^{\text {rd }}$ week ( $p=0.024)$ (Fig. 3).

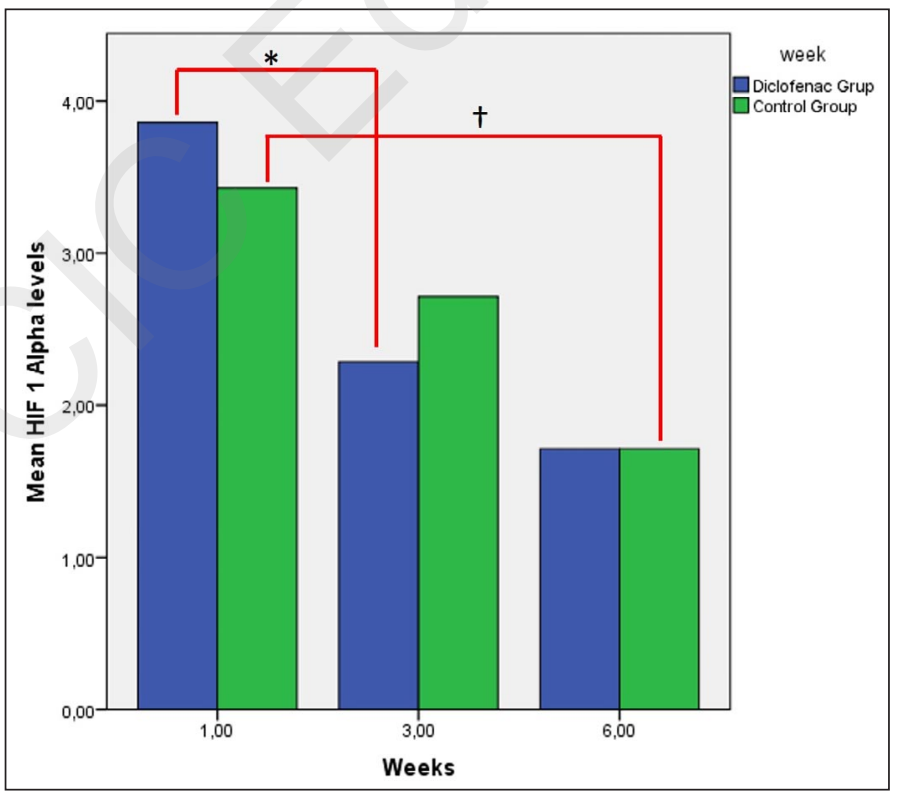

Figure 3. Changes in the mean HIF1a levels by the time in both groups.

* represents the significant decrease in the HIF1a at the $3^{\text {rd }}$ week and $\dagger$ the significant decrease in HIF1a levels at the $6^{\text {th }}$ week. 
There was a moderate correlation between HIF1a and vascular proliferation in control group $(p=0.008$ Rho $=0.559$ ) but this correlation was not observed in diclofenac group $(p=0.255$, Rho=0.260).

The vascular proliferation was highest at the $1^{\text {st }}$ week in the control and diclofenac group but was significantly low in the control group than diclofenac. Than vascular proliferation decreased in both groups and there was no significant difference between groups at $3^{\text {rd }}$ and $6^{\text {th }}$ weeks $(p=0.03, p=0.682, p=0.73$ respectively).

TNFa levels also were highest at the first week in both diclofenac and control group and decrease significantly at the $3^{\text {rd }}$ week in both groups $(p<0.001)$, but in the diclofenac group, TNFa level was high at $6^{\text {th }}$ week compared to $3^{\text {rd }}$ week $(p=0.05)$ (Fig. 4).

\section{Discussion}

Our key finding is that there is a difference in cytokine levels between the diclofenac group and control and the levels were decreased with time. One of the important finding of our study is HIF1a and TNFa levels were highest at the first week and significantly decreased by the weeks. In a rat tendon experiment model, HIF1a levels were analysed at $0,7,14$ and 28 days. HIF1a levels significantly increased immediately after surgery ( 1.53 fold) when compared to control group, then a significant decrease was observed at 14 and 28 days $^{19}$.

HIF1a is normally hydroxylated in presence of oxygen, iron and 2-oxoglutarate. After a trauma, a hypoxic environment occurs at the injured area. Hypoxia initiates intracellular stabilization of HIF1a.HIF1a reacts with von Hippel Lindau protein and then undergoes ubiquitination and destroyed. In hypoxia, the oxygen required for HIF1a to be ubiquitinated is missing. Thus HIF1a persists intact, moves to nucleus and recruits coactivator proteins to HIF binding site. The results are up-regulation of a large number of target genes and VEGF ${ }^{20}$. These genes play an important role in wound healing and tissue repair via pro-inflammatory, angiogenic and potentially profibrotic process ${ }^{21}$. So the highest of HIF1a levels with a correlation of vascular proliferation at the first week describes the inflammatory response.

Tendon healing is considered to occur in three overlapping phases: the inflammatory phase (1-11/2 weeks) the reparative/proliferative healing phase (1-6 weeks) and remodelling phase (4- weeks) ${ }^{22}$. In the inflammatory phase increase in angiogenesis plays as a key step in tissue regulation via providing oxygen and nutrients to the injured side. HIF1a is also recognised to control key aspects of inflammation, particularly leucocyte recruitment and subsequent cytokine production ${ }^{10}$. After preparation in the inflammatory phase, the reparative phase comes where the fibroblast takes the major role in tendon healing. In this phase naturally the HIF1a decreases because HIF1a shifts the cell metabolism from oxidative glycoxidation to glycolysis. If HIla persists in high concentrations, because of the build-up reactive oxygen species, cell death could be seen and thus could cause impairment of biomechanical properties ${ }^{23}$.

Altogether the facts indicate that environmental, also probably species dependent conditions and particular co-stimuli, are necessary to provoke pro- or antiapoptotic effects of TNFa in the tendon ${ }^{24}$. Tendon healing is probably influenced by the presence of proinflammatory cytokines such as TNFa. However tendon degeneration is also associated with increased TNFa in many studies, because tenocytes are highly activated by TNFa ${ }^{25}$. High levels of TNFa are secreted by activated macrophages and stimulate the downstream production of other pro-inflammatory cytokines such as interleukins 1, 6 and 8 and granulocyte-macrophage colony-stimulating factor by syn-

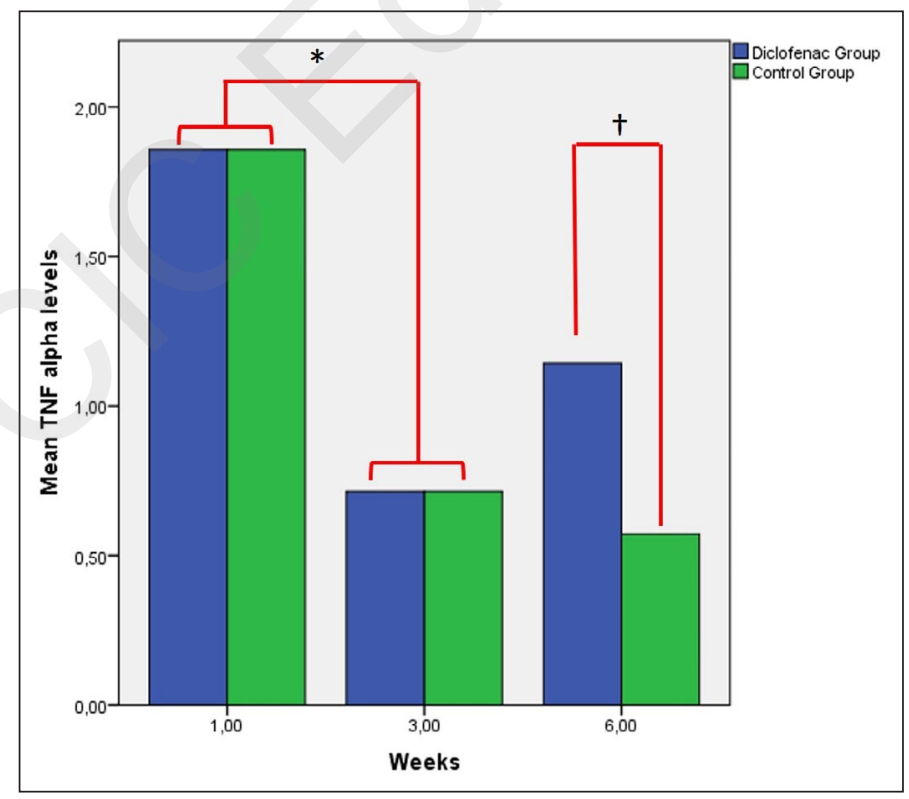

Figure 4. Changes in the mean TNFa levels by the time in both groups.

* represents the significant decrease of TNFa levels at $3^{\text {rd }}$ week in both diclofenac and control group and $†$ represents the significant difference in the $\mathrm{TN}$ a at $6^{\text {th }}$ week. 
ovial tissue via TNFR1 receptors. Also TNFa has TNFR2 receptors which modulate cell growth, differentiation and tissue repair ${ }^{9}$. The peak in the first week shows us the inflammatory phase of tendon healing. Rapid decrease at $3^{\text {rd }}$ week and steady state at $6^{\text {th }}$ week corresponds to reparative phase and may be a clue for tissue repair and cell growth modulation effect of TNFa.

In the diclofenac group, the HIF1a levels at the first week were higher than the control group. Also, because HIF1a up-regulates VEGF production, vascular proliferation was also high in diclofenac group at $1^{\text {st }}$ week $^{13}$. However there was a rapid decrease at $3^{\text {rd }}$ week in the diclofenac group. Because diclofenac has a potent anti-inflammatory effect this block in the inflammation may cause feedback effect on HIF1a and increase the HIF1a levels at the first week. But, when the healing phase progresses to reparative phase the HIF1a levels decrease rapidly. Diclofenac did not change TN a levels because while diclofenac inhibited cyclooxygenases and directed eicosanoid synthesis from prostaglandins towards leukotrienes, TNFa produced by macrophages, monocytes, fibroblasts and CD4+ T helper cells and played as a key driver of pro-inflammatory cytokine and prostaglandin E2 synthesis ${ }^{26}$. The interesting results were increased in TNF a levels from $3^{\text {rd }}$ to $6^{\text {th }}$ weeks. While HIF1a was high in the first week, the cells could shift their metabolism to glycolysis and resulting in an increase of free oxygen radicals. The magnitude and duration of hypoxic microenvironment and free oxygen radicals determines whether cells become apoptotic or adapt and survive. HiF1a is critical regulatory in this process $^{27}$. If microenvironment leads to necroptosis, which is a regulated form of necrosis, can trigger an innate immune response which could lead to increase in TNFa levels which could also impair the biomechanical properties of healed tendon ${ }^{28}$.

There are many limitations of this study. First, simulated tendon tear was acute tear rather than chronic tear. In chronic tears, TNFa seemed to play a major role in the tendon degeneration ${ }^{29}$. Second, we did not perform biomechanical testing. Although this is the main limitation of our study, in literature it is shown that inhibition of HIF prolyl 4 hydroxylases (a natural inhibitor of HIF1a in the body) improved mechanical properties of the enthesis ${ }^{30}$ and increased HIF1a IVEGF neoangiogenesis impaired biomechanical properties of the tendon ${ }^{19}$. These findings are compatible with our findings. Thirdly we did not obtain samples at day 0 which could be more precise as the baseline levels. But this is the first study that evaluates the HI1 a and TNFa levels in the acute setting of tendon repair and could put forth the possible effects of NSAIDs during healing via HIF1a and TNFa.

As a conclusion, HIF1a and TNFa both have a role in pro/antinflammatory process. They both play a role especially in the inflammatory process however HIF 1a levels also correlate with vascular proliferation in tendon healing. The HIF1a and TNFa levels were decreased as the time in normal tendon healing process. The diclofenac effects the levels of HIF1a and TNF a levels during this process.

\section{Compliance with ethical standards}

\section{Conflict of interest}

No other relationships/conditions/circumstances that present potential conflict of interest.

\section{References}

1. Galatz LM, Griggs S, Cameron BD, lannotti JP. Prospective longitudinal analysis of postoperative shoulder function: a tenyear follow-up study of full-thickness rotator cuff tears. J Bone Joint Surg Am. 2001;83-A(7):1052-1026.

2. Chona DV, Lakomkin N, Lott A, Workman AD, Henry AC Kuntz AF, et al. The timing of retears after arthroscopic rotator cuff repair. J Shoulder Elbow Surg. 2017;26(11)2054-2059.

3. Le BT, Wu XL, Lam PH, Murrell GA. Factors predicting rotator cuff retears: an analysis of 1000 consecutive rotator cuff repairs. Am J Sports Med. 2014;42(5):1134-1142.

4. Sharma P, Maffulli N. Biology of tendon injury: healing, modeling and remodeling. J Musculoskelet Neuronal Interact. 2006;6(2):181e90.

5. Kayaalp O. Non-Steroid anti inflamatory drugs. In: Kayaalp O (ed). Medical pharmacology, Hacettepe-Tas, Ankara. 2002:960-994.

6. Su B, O'Connor JP. NSAID therapy effects on healing of bone, tendon, and the enthesis. J Appl Physiol (1985). 2013 Sep;115 (6):892-899.

7. Tsai WC, Hsu CC, Chang HN, Lin YC, Lin MS, Pang JH. Ibuprofen upregulates expressions of matrix metalloproteinase $-1,-8,-9$ and -13 without affecting expressions of types I and III collagen in tendon cells. J Orthop Res. 2010;28 (4):487-491.

8. Brabcova E, Kolesar L, Thorburn E, Striz I. Chemokines induced in human respiratory epithelial cells by IL-1 family of cytokines. Folia Biol. 2014;60:180e6.

9. Innatko R, Kubes M. TNF signaling: early events and phosphorylation. Gen Physiol Biophys. 2007;26(3):159-167.

10. Cramer T, Yamanishi Y, Clausen BE, Förster I, Pawlinski R, Mackman N, et al. HIF-1alpha is essential for myeloid cell-mediated inflammation. Cell. 2003;7;112(5):645-657.

11. Yu Y, Zhou Y, Cheng T, Lu X, Yu K, Zhou Y, et al. Hypoxia enhances tenocyte differentiation of adipose-derived mesenchymal stem cells by inducing hypoxia-inducible factor- $1 \mathrm{a}$ in a coculture system. Cell Prolif. 2016;49(2):173-184.

12. Bernhardt WM, Wiesener MS, Weidemann A, Schmitt R, Weichert $\mathrm{W}$, Lechler $\mathrm{P}$, et al. Involvement of hypoxia-inducible transcription factors in polycystic kidney disease. Am J Pathol. 2007;170(3):830-842.

13. Hofer T, Desbaillets I, Höpfl G, Gassmann M, Wenger RH. Dissecting hypoxia-dependent and hypoxia-independent steps in the HIF-1alpha activation cascade: implications for HIF-1alpha gene therapy. FASEB J. 2001;15(14):2715-2717.

14. Bozova S, Elpek GO. Hypoxia-inducible factor-1alpha expression in experimental cirrhosis: correlation with vascular endothelial growth factor expression andangiogenesis. APMIS 2007;115(7):795-801.

15. Padulo J, Oliva F, Frizziero A, Maffulli N. Muscles, Ligaments and Tendons Journal - Basic principles and recommendations in clinical and field science research: 2016 update. MLTJ. 2016;6(1):1-5.

16. Cabuk H, Avci A, Durmaz H, Cabuk FK, Ertem F, Muhittin Şener I. The effect of diclofenac on matrix metalloproteinase levels in the rotator cuff. Arch Orthop Trauma Surg. 2014;134 (12): $1739-1744$

17. Fukuda H, Hamada K, Nakajima T, Tomonaga A. Pathology 
and pathogenesis of the intratendinous tearing of the rotator cuff viewed from en bloc histologic sections. Clin Orthop Relat Res. 1994;304:60-67.

18. Khandoga A, Enders G, Biberthaler P, Krombach F. Poly(ADP-ribose) polymerase triggers the microvascular mechanisms of hepatic ischemia-reperfusion injury. Am J Physiol Gastrointest Liver Physiol. 2002;283(3):G553-560.

19. Sahin H, Tholema N, Petersen W, Raschke MJ, Stange R. Impaired biomechanical properties correlate with neoangiogenesis as well as VEGF and MMP-3 expression during rat patellar tendon healing. J Orthop Res. 2012;30(12): 1952-1957.

20. West JB. Physiological Effects of Chronic Hypoxia. N Engl J Med. 2017;18;376(20):1965-1971.

21. Wang GL, Jiang BH, Rue EA, Semenza GL. Hypoxia-inducible factor 1 is a basic-helix-loop-helix-PAS heterodimer regulated by cellular $\mathrm{O} 2$ tension. Proc Natl Acad Sci USA. 1995;6;92(12):5510-5514.

22. Ackermann PW, Calder J, Aspenberg P. Healing and repair mechanism. In: Achilles tendon rupture. Current concepts, DJO publications, Guildford, UK. 2008:17-26.

23. Semenza GL. Hypoxia-inducible factors in physiology and medicine. Cell. 2012;3;148(3):399-408.
24. Schulze-Tanzil G, Al-Sadi O, Wiegand E, Ertel W, Busch C, $\mathrm{Kohl} \mathrm{B}$, et al. The role of pro-inflammatory and immunoregulatory cytokines in tendon healing and rupture: new insights. Scand J Med Sci Sports. 2011;21(3):337-351.

25. John T, Lodka D, Kohl B, Ertel W, Jammrath J, Conrad C, et al. Effect of pro-inflammatory and immunoregulatory cytokines on human tenocytes. J Orthop Res. 2010;28(8):1071-1077.

26. Feldmann M, Maini RN. Anti-TNF alpha therapy of rheumatoid arthritis: what have we learned? Annu Rev Immunol. 2001; 19:163-196.

27. Nathan C. Points of control in inflammation. Nature. 2002;1926;420(6917):846-852.

28. Newton K, Manning G. Necroptosis and Inflammation. Annu Rev Biochem. 2016;2;85:743-763.

29. Hosaka Y, Kirisawa R, Ueda H, Yamaguchi M, Takehana K. Differences in tumour necrosis factor (TNF)alpha and TNF receptor-1-mediated intracellular signalling factors in normal, inflamed and scar-formed horse tendons. J Vet Med Sci. 2005;67(10):985-991.

30. Gumucio JP, Flood MD, Bedi A, Kramer HF, Russell AJ, Mendias CL. Inhibition of prolyl 4-hydroxylase decreases muscle fibrosis following chronic rotator cufftear. Bone Joint Res. 2017;6(1):57-65. 